\title{
An Epidemiologic Approach to the Development of Early Trauma Focused Intervention
}

\author{
Douglas F. Zatzick \\ Department of Psychiatry \& Behavioral Sciences, Harborview Medical Center, \\ University of Washington School of Medicine, Seattle, WA \\ Sandro Galea \\ University of Michigan School of Public Health, Ann Arbor, MI and Columbia University \\ Mailman School of Public Health, New York City, NY
}

\begin{abstract}
Early, trauma-focused intervention development has emphasized unidirectional trajectories that begin with basic research and efficacy trials followed later by effectiveness and dissemination studies. In this article, the authors present methods derived from social and clinical epidemiology that constitute foundational research in the development of early trauma-focused intervention. They also describe how population-based practice research may serve to feed back and inform what has been conceptualized as earlier stages of intervention development such as efficacy trials. Examples of relevant epidemiologic research methods are presented to illustrate these points. The authors posit that the continued application of population-based methods may produce treatments that can be feasibly applied to the unique patient, provider, organizational, and community contexts relevant to early interventions for survivors of trauma.
\end{abstract}

Psychopharmacological and psychotherapeutic intervention development has often been conceptualized as moving along a continuum from basic research through efficacy trials to investigations that test intervention effectiveness and ultimately widespread dissemination (Holder et al., 1999; Rounsaville, Carroll, \& Onken, 2001; Weisz, Chu, \& Polo, 2004). To date, this bench-to-bedside conceptualization of treatment development may be seen as the predominant framework informing early trauma-focused interventions (Davis, Barad, Otto, \& Southwick, 2006; Foa \& Meadows, 1997; Litz, Gray, Bryant, \& Adler, 2002; National Institute of Mental Health, 2002). Other commentary has suggested that diverse investigative designs that vary across efficacy, effectiveness, and dissemination/practice research paradigms may optimally inform the development of interventions that are feasibly and effectively delivered in real-world settings (Kazdin, 2001; National Institute of Mental Health, 1999; SouthamGerow, Ringeisen, \& Sherrill, 2006; Street, Niederehe, \& Lebowitz, 2000; Weisz et al., 2004; Zatzick, Simon, \& Wagner, 2006). There exists, however, a paucity of discussion in the peer-reviewed literature of how diverse methodological approaches can inform the development of early, trauma-focused intervention. The current overview

\footnotetext{
This research was supported by National Institute of Health grants MH 073613, DA 017642, and MH 078152 . A preliminary version of this manuscript was presented at the 22nd Annual Meeting of the International Society of Traumatic Stress Studies, Los Angeles, California, November 6, 2006.

Correspondence concerning this article should be addressed to: Douglas F. Zatzick, Department of Psychiatry \& Behavioral Sciences, Harborview Medical Center, University of Washington, PO Box 359911, 325 9th Avenue, Seattle, WA 98104-2499. E-mail: dzatzick@u.washington.edu. 


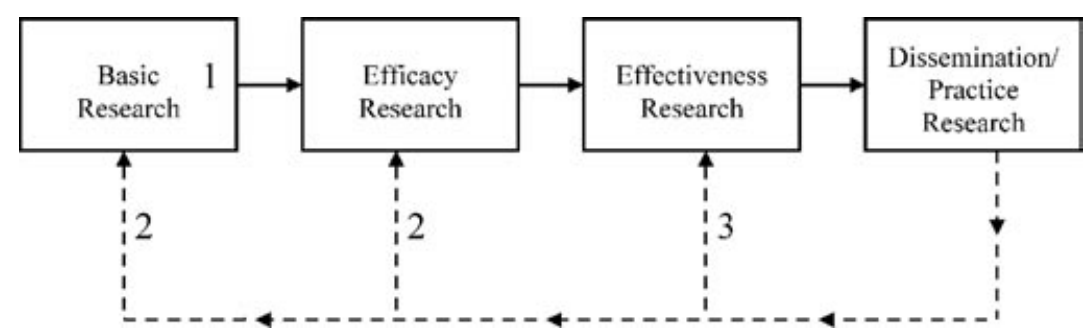

Figure 1. The contribution of epidemiological studies to early intervention development. $1=$ Basic investigation of variations in posttraumatic biological parameters. $2=$ Practice research informs earlier phases of intervention development. $3=$ Epidemiological studies inform combined, stepped-care interventions. Practice research is defined as investigation that examines how and which treatments or services are actually provided to individuals within real-world service delivery contexts (National Institute of Mental Health, 1999). Adapted from Bridging Science and Service: A Report by the National Advisory Mental Health Council's Clinical Treatment and Services Research Workgroup (NIH Publication No. 99-4353), by National Institute of Mental Health, 1999, Bethesda, MD: National Institute of Mental Health.

addresses this gap by providing one conceptual framework for and examples of how population-based epidemiological investigation can contribute to the development of early, trauma-focused interventions (Figure 1).

\section{EPIDEMIOLOGIC METHODS AND INTERVENTION DEVELOPMENT}

Broadly speaking, epidemiology is the science that describes the distribution of diseases and attempts to elucidate associations between disease determinants and specific disease states (Rothman \& Greenland, 1998). Prior commentary has conceptualized epidemiologic research as a basic science in the development of medical and psychosocial interventions (Greenwald, 1984; Holder et al., 1999; Shrout, 1998). Epidemiology, as a foundational science for intervention development, can serve to define the incidence and prevalence of specific disorders and aid in the establishment of working causal models of a disorder, including the elucidation of risk and protective factors (Holder et al., 1999). We note here that the role of epidemiology in identifying causes of disease has been the subject of substantial debate in the literature (Kundi, 2006; Susser, 1997). We use here the more general word determinant (Susser, 1991) to refer to factors that are statistically demonstrated to be associated with health indicators of interest, taking no side in the debates about whether epidemiologic inquiry can genuinely understand causality, or identify causes.

In the past few decades, as epidemiology has matured as a science, different branches of epidemiology have emerged. Psychiatric epidemiology, as one of these branches, is concerned with the study of the distribution and determinants of psychiatric disorders in the general population (Galea, Nandi, \& Vlahov, 2005; Insel \& Fenton, 2005). One of the key challenges with which epidemiologists have grappled during the past decade is the scope of the determinants that constitute areas of interest and focus for epidemiology. This has often been referred to as epidemiology that is concerned with levels of etiology and inquiry. For many of the early years of epidemiology's formation as a discipline, the central focus of epidemiologic research interest was on the individual level and individual risk factors for diseases. These risk factors were primarily individual behaviors or environmental exposures that affected individuals. Recently, however, there has been growing interest in pushing the boundaries of epidemiologic inquiry beyond the individual, to include an individual's context, such as characteristics of communities of residence and interactions with the health care system. Social epidemiology has evolved as a specific area of epidemiology focusing on context and the social 
determination of health and disease, although, broadly speaking and relevant to this discussion, social epidemiology offers a lens through which all disease causation can be understood. Therefore, for this discussion, we refer to social/psychiatric epidemiology to mean etiologic population-based inquiry that is concerned with causation at multiple levels.

In contrast, clinical epidemiology can be understood as the science of making predictions about individual patients by describing clinical phenomena (e.g., psychiatric symptoms, medical comorbidities) in populations of patients (Fletcher, Fletcher, \& Wagner, 1996). Clinical epidemiology aims to frame the care of the individual patient in the context of the larger population of patients that present for care in a specified health service delivery setting.

Social and clinical epidemiology share in common a multifactorial approach to the understanding of disease causality and amelioration (Rothman \& Greenland, 1998). Epidemiologic approaches to understanding causal associations emphasize the examination of a broad spectrum of evidence, including the strength of an association, identification of a temporal relationship in which the determinant precedes the disorder, consistency in the repeated observation of an association across different populations, specificity with regards to a single determinant leading to a single effect, observation of a biological gradient or doseresponse relationship between determinant and effect, biological plausibility, coherence in the sense that what is known about the association does not conflict with what is known about the disease process, analogy to disease processes for other disorders, and experimental evidence supporting the association or amelioration of the condition when a hypothesized determinant is targeted in randomized clinical trial designs (Chilcoat \& Breslau, 1998; Rothman \& Greenland, 1998). Reliance upon this spectrum of information to elucidate causal associations has necessitated that social and clinical epidemiologic investigators be familiar with a range of investigative designs that include randomized clinical trials as well as cross-sectional and prospective cohort designs (Feinstein, 1985; Rothman \& Greenland, 1998).
Epidemiology is a population-based science; therefore, inference is drawn from studies among population groups and about population aggregate risks and rates. Both social and clinical epidemiological investigations strive to attain external validity through representative sampling procedures that emphasize the inclusion of a quantifiable denominator in the investigative design. Social/psychiatric epidemiology and clinical epidemiology can be distinguished by thinking through the question, "What is the denominator?” In classic psychiatric epidemiology the ideal denominator is the general population (Kessler, Sonnega, Bromet, Hughs, \& Nelson, 1995; Wang et al., 2005). For trauma-focused psychiatric epidemiology, the entire trauma-exposed population often constitutes the denominator (Galea et al., 2002; Hoge, Auchterlonie, \& Milliken, 2006; Kulka et al., 1990; Norris et al., 2002). This is illustrated in Figure 2 as all individuals within a trauma-exposed region. For clinical epidemiology, the denominator can be defined by a population of patients that present to a particular clinical setting (Feinstein, 1985). For example, clinical epidemiological studies of injured trauma survivors conducted in acute care medical settings define the population of patients admitted to a trauma center during the time period of the study as the denominator (Zatzick, Grossman et al., 2006; Zatzick et al., 2004). This is illustrated in Figure 2 by the encapsulation of individuals within health care organizations. In studies of health care systems, the denominator may include all organizations within a particular geographic region (Figure 2; Mackenzie et al., 2006; Zatzick et al., 2007).

Barriers in the widespread dissemination of evidencebased practice to real-world treatment settings may arise from aspects of the intervention development processes that fail to adequately take the influence of contextual factors on treatment implementation into consideration (Glasgow \& Emmons, 2007; Zatzick, Simon, et al., 2006). A central difficulty may be the lack of focus on issues of external validity up-front in the research design. As a result, investigations establishing the efficacy of interventions may suffer from the inclusion of nonrepresentative samples of patients, providers, and health care organizations (Spinazzola, Blaustein, \& van der Kolk, 2005). 
Trauma Exposed Region
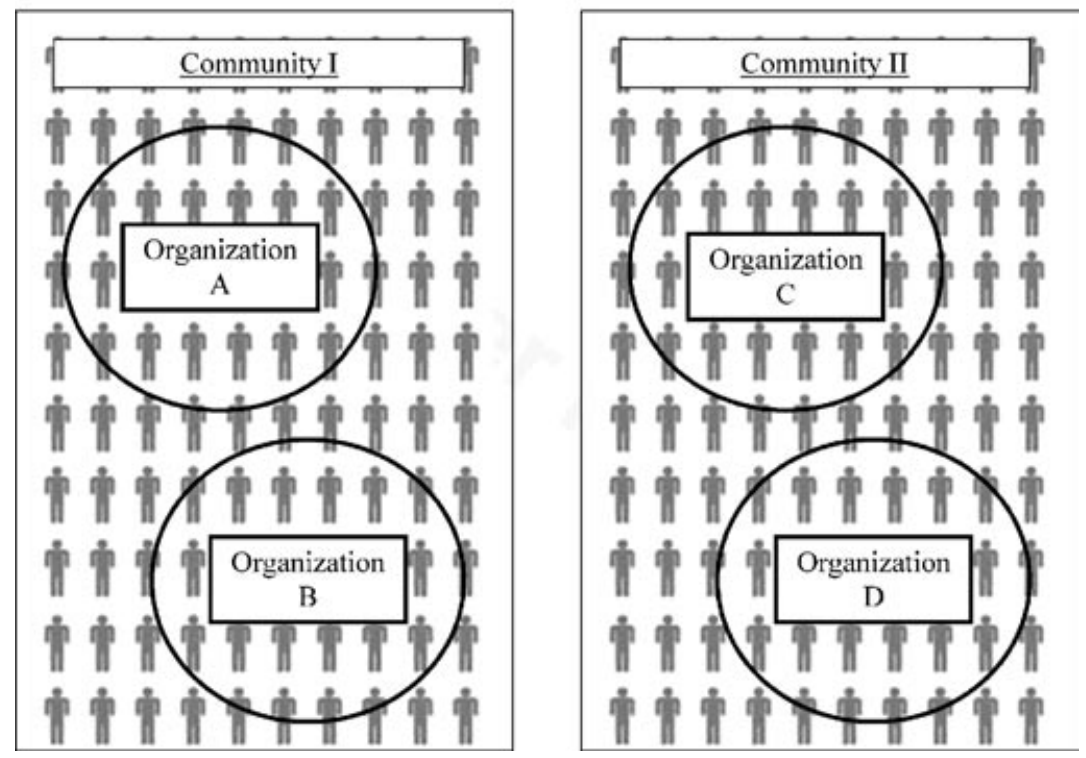

Figure 2. An example of multiple potential denominators in a trauma-focused social and clinical epidemiologic investigation. The first denominator is defined as all individuals within a trauma-exposed region. A second denominator might consist of the subpopulation of individuals within a single trauma exposed community (e.g., individuals in Community I). A third potential denominator is all trauma-exposed individuals presenting to a single health care organization, such as a level I trauma center (e.g., individuals presenting to organization $\mathrm{A}$ ). A fourth denominator could be defined as all health care organizations within a trauma exposed region (e.g., Organizations A, B, C, and D).

Glasgow and Emmons (2007) have articulated a multifactorial model of the types of evidence required for a comprehensive understanding of contextual factors in the translation of efficacious interventions to real-world treatment settings. Theoretical rationale/mechanism of action and efficacy data are two crucial domains of evidence required. Beyond these domains, information regarding external validity/generalizability, including data on the feasibility of intervention delivery in representative practice settings, cost, and economic data, and ongoing quality improvement/safety information may also be required for successful widespread dissemination.

We posit that population-based epidemiological investigation is an ideal method for systematically obtaining patient, provider, organizational, and community contextual data required for the development of robustly delivered early, trauma-focused interventions. In the next section, we provide specific case examples of how social and clinical epidemiological investigations constitute basic research on intervention development and how practice and dissemination research clearly feed back to inform what has been previously conceptualized as earlier stages of intervention development (Figure 1). The examples we consider here discuss how representative samples can be invaluable in investigations concerned with the genetic/molecular determinants of psychopathology, pharmacologic prevention of posttraumatic stress disorder (PTSD), and early PTSD intervention trials. We refer to specific pathways and elements from Figure 1 throughout our discussion of each of these examples. 


\section{EXAMPLES OF THE POTENTIAL CONTRIBUTIONS OF SOCIRL AND CLINICAL EPIDEMIOLOGY TO THE DEVELOPMENT OF ERRLY, TRAUMA-FOCUSED INTERVENTION}

\section{Example 1. Population Sampling and Genetic Determinants of Psuchopathology}

Traditionally, etiologic research that is concerned with the determination of particular psychopathologies is conducted among highly selected (and predominantly homogenous) groups of persons enrolled in clinical settings (see Figure 1, Pathway 1; Galea et al., 2006). The principal rationale for using such highly selected groups for the purposes of genetic/molecular inquiry is that having homogenous persons enrolled in a particular study minimizes interperson variation and the confounders that such variation may introduce, complicating the particular gene/molecule and disease association of interest. However, this approach has the potential to obscure important insight about the etiologic mechanisms that operate in influencing pathogenesis. As discussed above, epidemiologists have articulated multifactorial causal formulations. From this perspective, much of what we may typically think of as a cause for disease is actually a component cause of a larger sufficient cause. It is the constellation of component causes that make up a sufficient cause for pathogenesis. There are many (perhaps an infinite number) of sufficient causes of any particular disease, each constituted of their own component causes. This heuristic has the advantage of explaining why it is that persons with a particular cause do not always have disease. Consider component cause X, which is a wellrecognized risk factor for disease $\mathrm{Y}$. Although component cause $\mathrm{X}$ may be a part of many sufficient causes for disease $\mathrm{Y}$ (hence the presence of that component cause X conferring high risk on a person for disease etiology), the absence of the other component causes that need to co-occur with component cause $\mathrm{X}$ may mean that a particular individual does not develop disease Y. Conversely, the presence of even a few sufficient causes that do not involve component cause $\mathrm{X}$ means that persons without cause $\mathrm{X}$ (persons at low risk because there are only a few sufficient causes that do not involve X) can still develop disease Y. This heuristic can then help us understand why population-based epidemiologic methods are necessary if we want to understand the true nature of genetic/molecular determination of disease. To illustrate this, let us assume that we can produce a particular psychopathology of interest $\mathrm{P}$ in three ways (and only three ways); i.e., there are three sufficient causes of PTSD. And let us also assume that these sufficient causes are as follows:

- Sufficient Cause 1: Requires the co-occurring presence of factors G1, factor S1, and factor U. Let us assume that $\mathrm{G} 1$ is a particular gene, $S 1$ is a particular social factor and $\mathrm{U}$ is the set of all other unmeasured factors.

- Sufficient Cause 2: Following the same notation as above, requires the co-occurring presence of factors G2 (another particular gene), and factor U.

- Sufficient Cause 3: Requires the co-occurring presence of only factors S2, another particular social factor and factor $U$.

Now suppose that each of these sufficient causes are present in a third of the population; that is, that a third of the population has factors $\mathrm{G} 1, \mathrm{~S} 1$, and $\mathrm{U}$, a third have $\mathrm{G} 2$ and $\mathrm{U}$, and a third have S2 and U. If we were to then conduct a study that selects from a narrowly defined group of persons (e.g., persons from a local emergency department) we may select only persons who have factors G2, S2, and U; it is entirely possible (especially if our recruitment is highly selective) that we do not find anyone with factors $S 1$ or factors $\mathrm{G} 1$. In so doing, we may then have disease causation predicated on causes 2 and 3 only, and we may well conclude that factors G2 (i.e., a genetic factor) or factor S2 (i.e., a social factor) are both causes of PTSD, but that there is no biologic interaction between genetic or social factors in the cause for PTSD. Now, conversely, if in a different sample, we select people who only happen to have factors G1 and S1, we may well not even notice that these factors are component causes for PTSD because they are ubiquitous and hence there is no variability in relation between exposure (constellation of sufficient causes) and outcome. Both these inferences would of course be false. 
The truth is that there are multiple avenues of causation for disease $P$, including interaction between factors G1 and factor $S 1$, but that this interaction is not detected in either scenario when we failed to sample persons in whom the interaction exists, or to sample in such a way that we notice that there are indeed multiple causal genes that are not sampled in a particular homogenous sample. Therefore, population-based sampling can provide an opportunity to much more accurately elucidate the joint contribution of genetic/molecular and social causes to disease etiology.

\section{Example 2. Epidemiologic Investigation of Psuchiatric Comorbidities Informs Early Combined Intervention}

There is an abundance of epidemiologic evidence that PTSD co-occurs with other mood, anxiety, and substance related comorbidities (Hoge et al., 2004; Kessler et al., 1995; Kulka et al., 1990). Galea and colleagues' population-based investigations after the September 11, 2001 World Trade Center terrorist attack documented high frequencies of PTSD symptoms as well as comorbid depressive symptoms and increases in alcohol and tobacco use (Boscarino, Adams, \& Galea, 2006; Galea et al., 2002; Vlahov, Galea, Ahern, Resnick, Boscarino, et al., 2004; Vlahov, Galea, Ahern, Resnick, \& Kilpatrick, 2004). For example, this series of studies found that the prevalence of drinking problems was $3.7 \%$ in the 6 months before September 11 and $4.2 \%$ in the 6 months after September 11. The incidence of drinking problems among those without drinking problems before September 11 was 2.2\%. Persons with incident drinking problems were more likely than those without to report symptoms consistent with PTSD (17.4\% vs. $0.4 \%$ in those without drinking problems and $1.4 \%$ in nondrinkers) and depression (23.5\% vs $5.6 \%$ vs. $4.9 \%$, respectively) after September 11, 2001 (Vlahov et al., 2006). See Figure 1, Pathways 1 and 3 (Galea et al., 2002; Vlahov, Galea, Ahern, Resnick, Boscarino et al., 2004; Vlahov, Galea, Ahern, Resnick, \& Kilpatrick, 2004; Zatzick et al., 2004).

These data are consistent with findings in other disasterexposed populations. For example, Reijneveld, Crone,
Verhulst, and Verloove-Vanhorick (2003) found PTSD, depressive, and alcohol use symptoms were elevated among Dutch adolescents exposed to a café fire. Interestingly, as with the September 11, 2001 terrorist attacks, longterm follow-up demonstrated sustained elevations in alcohol use, while PTSD and depressive symptoms diminished (Reijneveld, Crone, Schuller, Verhulst, \& VerlooveVanhorick, 2005). An epidemiologic approach to early intervention development might use these findings as foundational research to inform the development of early combined interventions targeting not only PTSD, but also highly prevalent mood and substance-related morbidities.

An important corollary to this observation is that epidemiological investigation may also incorporate measures of the public health burden of specific disorders, such as the population attributable fraction (PAF), attributable years of life lost, and disability adjusted life years, to fully describe the burden of disease that attends traumatic event exposure and to more fruitfully inform the evaluation of combined intervention procedures (Steenland \& Armstrong, 2006). For example, evidence suggests that PTSD may be a risk factor for a variety of chronic medical conditions (Schnurr \& Green, 2004). Also, alcohol misuse in trauma-exposed populations may be associated with additional morbidity/mortality secondary to recurrent traumatic injury (Gentilello et al., 1999; Hearst, Newman, \& Hulley, 1986). Thus, population-based prospective cohort investigations that incorporate PAF estimates for PTSD and related comorbidities could inform the staging and sequencing of early combined intervention procedures that target the primary and secondary prevention of PTSD and related comorbidities.

\section{Example 3. Practice Research Informs Basic and Efficacu Research in Secondaru PTSD Prevention}

The theoretical rationale for a diverse group of candidate compounds as early intervention agents in the secondary psychopharmacologic prevention of PTSD has been articulated (Friedman, 2002; Morgan, Krystal, \& Southwick, 2003; Pitman \& Delahanty, 2005; Schoenfeld, Marmar, 
\& Neylan, 2004). Among these potential agents, corticosteroids and beta-adrenergic antagonists have recently been selected for initial efficacy trials (Pitman et al., 2002; Schelling et al., 2004; Stein, 2005; Vaiva et al., 2003). See Figure 1, Pathway 2 (Zatzick \& Roy-Byrne, 2006).

Preventive pharmacotherapeutic intervention targeting PTSD is hypothesized to begin optimally early after the traumatic event (Friedman, 2002; Pitman \& Delahanty, 2005). At the time of surgical inpatient discharge, injured patients are typically off intravenously administered medication and follow-up care is being planned. Thus, hospital discharge may be a key time point to initiate medications targeting the secondary prevention of PTSD among injured trauma survivors (Zatzick \& Roy-Byrne, 2006).

However, delivery of interventions in acute care is complicated by a number of patient, provider, and service delivery contextual factors that, when explored through population-based investigation, may serve to inform which of the many medications with strong theoretical rationales for secondary PTSD prevention can be feasibly delivered early on posttrauma. Data from 2,931 patients recruited from 69 acute care inpatient hospitals nationwide suggests that, along with early posttraumatic distress, physical pain complaints within 2-3 months postinjury are significantly and independently associated with an increased risk of symptoms consistent with a diagnosis of PTSD 12 months after injury hospitalization (Zatzick et al., 2007). Another population-based clinical phenomenological investigations has demonstrated that patients are predominantly concerned with physical health and bodily pain in the days and weeks after injury; psychological concerns such as anxiety and depressive symptoms are less prevalent within the days after the injury but steadily increase over the ensuing months (Zatzick et al., 2001; Zatzick et al., 2007).

These observations informed a pharmacoepidemiologic investigation of medication prescription by surgical providers at the time of acute care hospital discharge. Medication prescription at the time of hospital discharge for randomly selected adolescent $(n=113)$ and adult $(n=152)$ injury survivors was assessed by review of automated medical records. Opiate analgesics were prescribed for $80 \%-$ $90 \%$ of patients, and nonopiate analgesics to $34 \%-46 \%$ of patients. Corticosteroids, beta-adrenergic blockers, and other psychotropic medications were prescribed to less than $10 \%$ of patients (Zatzick \& Roy-Byrne, 2006).

The results of this pharmacoepidemiologic investigation substantiate the ubiquitous use of analgesic medication in the acute care inpatient setting. These data, when taken into consideration with preclinical data suggesting opiates may prevent memory consolidation through a betaadrenergic mechanism (McGaugh, Introini-Collison, \& Nagahara, 1988; Morgan et al., 2003), suggest initial feasibility tests and efficacy trials of compounds targeting pain in the secondary prevention of PTSD after injury. These results might also stimulate basic research on compounds that simultaneously target pain and anxiety. The findings demonstrate how population-based data derived from realworld practice settings can enhance the efficiency and trajectories of pharmaceutical intervention development in the secondary prevention of PTSD.

\section{Example 4. How Population-Based Sampling Can Enhance the External Validity of Early Intervention Trials}

Randomized controlled trials have established the efficacy of psychotherapeutic and psychopharmacological treatments in the treatment of posttraumatic stress disorder (PTSD) and related comorbid conditions, and guidelines derived from these studies have been developed (Department of Veterans Affairs, 2003; Foa, Keane, \& Friedman, 2000; Ursano et al., 2004). Despite these advances many individuals either go untreated or do not receive guideline-concordant PTSD care (Katon, Zatzick, Bond, \& Williams, 2006).

A key issue for the development of early, trauma-focused intervention is that treatments derived from efficacy trials developed in mental health specialty settings may require adaptation to be feasibly delivered in real-world early intervention settings. For example, the standardized regular appointments and intact health service delivery systems that constitute an implicit foundation of treatment delivery in efficacy trials are often impossible to attain in 
early posttraumatic treatment contexts such as postdisaster or acute care medical settings (Sabin, Zatzick, Jurkovich, $\&$ Rivara, 2006). Also, population-based clinical investigation suggests that, in contrast to the highly motivated patients recruited into efficacy trials, patients in the early aftermath of trauma experience multiple other posttraumatic concerns that extend beyond seeking treatment for PTSD (Zatzick et al., 2001; Zatzick et al., 2007). The process of adaptation might necessarily begin with social and clinical epidemiological investigations that characterize the early posttraumatic individual, provider, organizational, and community contexts relevant to the implementation of early, trauma-focused interventions.

One manner in which the acute care traumatic injury research programs have operationalized an epidemiological approach to intervention development is through the use of population-based automated data systems (Simon, Unutzer, Young, \& Pincus, 2000; Zatzick et al., 2000). The population-based automated data systems provide clinical and demographic information on all patients treated within the acute care inpatient setting so that characteristics of an individual patient or subgroup of patients included in an investigation can be compared to the population of patients presenting for care (Figure 2).

Typically, a series of prospective cohort and crosssectional investigations leads to randomized trials. These preparatory investigations use the data systems (in conjunction with structured clinical assessments) to gain insight into the processes of care underlying the detection of patients with mental health symptoms/diagnoses (Sabin et al., 2006; Zatzick et al., 2005), to characterize the symptomatic presentation and trauma histories of patients to be targeted in a clinical trial (Zatzick, Grossman, et al., 2006; Zatzick et al., 2002), and to characterize population utilization and cost parameters (Zatzick et al., 2000). The automated data systems can also provide key data related to policy-relevant outcome domains, such as emergency department and inpatient surgical surveillance data documenting recurrent injury admissions (Gentilello et al., 1999).

With regard to the actual conduct of randomized clinical trials, the automated data systems allows the first portion of the results section of the trial to ask how the clinical and demographic characteristics of individuals included in the investigation compare to the population of eligible patients admitted to the trauma center during the time period of the study. The first table or paragraph of the manuscript's results section compares the clinical, injury, and demographic characteristics of participating patients with the characteristics of the population of patients admitted to the trauma center (Zatzick, Grossman, et al., 2006; Zatzick et al., 2002; Zatzick et al., 2004). Overall, the clinical, demographic, and injury characteristics of patients included in the acute care prospective and randomized clinical trials investigations have not significantly differed from the characteristics of the injured population, with the exception of inpatient length of stay.

In an effort to understand bias introduced by longer inpatient stays in study patients, other trauma registry investigations have assessed the characteristics of patients who are discharged early from the hospital (Zatzick et al., 2000). In a trauma registry investigation of over 10,000 inpatient admissions, patients with alcohol abuse/dependence diagnoses demonstrated a $10 \%$ reduction in inpatient length of stay; thus, patients with these diagnoses may be underrepresented in the study samples. In summary, the use of automated trauma registry data allows for the assessment of generalizability and bias in acute care inpatient clinical trial sampling.

\section{CONCLUSIONS}

The four examples presented demonstrate how the population-based approaches of social and clinical epidemiology can productively contribute to the development of early, trauma-focused interventions. Contributions can occur at multiple levels, including foundational research that informs intervention development and also redirection of treatment development trajectories towards more efficiently and robustly applied real-world interventions.

Pioneers in key fields that have informed the development of trauma-focused intervention such as clinical psychology have been equally concerned with issues of external 
validity (Bronfenbrenner, 1977; Campbell, 1957; Mook, 1983). Clinical psychologists have raised questions about the extension of data derived from experimental designs to social settings (Campbell, 1957) and regarding the ecological validity of experimental findings (Bronfenbrenner, 1977, 1979). Public health approaches to the development of early, trauma-focused interventions would suggest that to develop ecologically valid interventions, external validity/generalizability assessments should be more fastidiously incorporated upfront into the design and implementation of clinical investigations (Glasgow \& Emmons, 2007; Zatzick, Simon, et al., 2006).

The approaches presented here aim to systematically incorporate considerations regarding patient, provider, organizational, and community context into the development and implementation of early, trauma-focused interventions (Figure 2). The use of population-based sampling procedures and registry information systems operationalizes methods to enhance assessments of generalizability.

We recognize that encouraging early intervention researchers to develop the methodological expertise required for population-based investigation may be arduous. Although there have been increasing calls for such work, relatively few studies have yet been carried out that use population samples to consider questions related to biologic etiology (Cacioppo, Berntson, Sheridan, \& McClintock, 2000; Galea et al., 2006). We suspect that the principal barrier to the establishment of such studies is not so much cost, as the technical and intellectual flexibility to implement such studies. Solutions, however, are emerging. Population-based automated data analyses on existing information systems, such as the pharmacoepidemiolgical investigation described in Example 3 are remarkably inexpensive. An approach that may overcome barriers to developing needed methodological expertise is the cultivation of interdisciplinary teams that augment research groups comprised primarily of psychologists, psychiatrists, and other mental health investigators with collaborators from diverse social scientific (e.g., economics, anthropology), medical (e.g., internal medicine, pediatrics, surgery), and public health backgrounds (Pellmar \& Eisenberg, 2000; Zatzick, Simon, et al., 2006). As discussed here, incorporation of these methods promises to advance early-intervention science substantially.

It is well established that the causes of psychopathology after traumatic exposure are multifactorial. However, considering only one factor (in this case traumatic exposure) as central to early intervention, absent of a full consideration of the role of context, has produced treatments that do not broadly generalize to trauma-exposed populations. Ultimately, interventions that are predicated on a restricted sampling frame may hold diminished relevance when applied to broader real world contexts. Although a well-designed randomized efficacy trial may demonstrate a large effect size in a homogenous sample, the intervention may need augmentation if it is to be broadly implemented with the heterogeneous populations that characterize realworld early intervention settings. Ultimately, the multifactorial influence of context stacks the deck against interventions informed only by one risk factor and suggests results that, in a practical sense, are unattainable. We hope that the approaches proposed here-applying population-based methods to understand fully the unique and relevant patient, provider, organizational, and community contextsmay help in the development of feasibly applied, effective, early interventions to mitigate the multiple adverse consequences of individual and mass trauma.

\section{REFERENCES}

Boscarino, J. A., Adams, R. E., \& Galea, S. (2006). Alcohol use in New York after the terrorist attacks: A study of the effects of psychological trauma on drinking behavior. Addictive Behaviors, 31, 606-621.

Bronfenbrenner, U. (1977). Toward an experimental ecology of human development. American Psychologist, 32, 513531.

Bronfenbrenner, U. (1979). The ecology of human development: Experiments by nature and design. Cambridge, MA: Harvard University Press.

Cacioppo, J. T., Berntson, G. G., Sheridan, J. F., \& McClintock, M. K. (2000). Multilevel integrative analyses of human behavior: Social neuroscience and the complementing nature of social and biological approaches. Psychological Bulletin, 126, 829843. 
Campbell, D. T. (1957). Factors relevant to the validity of experiments in social settings. Psychological Bulletin, 54, 297-312.

Chilcoat, H. D., \& Breslau, N. (1998). Investigations of causal pathways between PTSD and drug use disorders. Addictive Behaviors, 23, 827-840.

Davis, M., Barad, M., Otto, M., \& Southwick, S. (2006). Combining pharmacotherapy with cognitive behavioral therapy: Traditional and new approaches. Journal of Traumatic Stress, 19, 571-581.

Department of Veterans Affairs. (2003). Management of post-traumatic stress. Retrieved April 17, 2005, from http://www.oqp.med.va.gov/cpg/PTSD/PTSD_Base.htm

Feinstein, A. R. (1985). Clinical epidemiology: The architecture of clinical research. Philadelphia: W. B. Saunders Co.

Fletcher, R. H., Fletcher, S. W., \& Wagner, E. H. (1996). Clinical epidemiology: The essentials (3rd ed.). Baltimore, MD: Williams and Wilkins.

Foa, E. B., Keane, T. M., \& Friedman, M. J. (2000). Guidelines for treatment of PTSD. Journal of Traumatic Stress, 13, 539-588.

Foa, E. B., \& Meadows, E. A. (1997). Psychosocial treatments for posttraumatic stress disorder: A critical review. Annual Review of Psychology, 48, 449-480.

Friedman, M. J. (2002). Future pharmocotherapy for post-traumatic stress disorder: Prevention and treatment. Psychiatric Clinics of North America, 25, 427-441.

Galea, S., Acierno, R., Ruggiero, K., Resnick, H., Tracy, M., \& Kilpatrick, D. (2006). Social context and the psychobiology of posttraumatic stress. Annals of the New York Academy of Sciences, 1071, 231-241.

Galea, S., Ahern, J., Resnick, H., Kilpatrick, D., Bucuvalas, M., Gold, J., et al. (2002). Psychological sequelae of the September 11 terrorist attacks in New York City. New England Journal of Medicine, 346, 982-987.

Galea, S., Nandi, A., \& Vlahov, D. (2005). The epidemiology of post-traumatic stress disorder after disasters. Epidemiologic Reviews, 27, 78-91.

Gentilello, L. M., Rivara, F. P., Donovan, D. M., Jurkovich, G. J., Daranciang, E., Dunn, C. W., et al. (1999). Alcohol interventions in a trauma center as a means of reducing the risk of injury recurrence. Annals of Surgery, 230, 473-480.

Glasgow, R. E., \& Emmons, K. M. (2007). How can we increase translation of research into practice? Types of evidence needed. Annual Review of Public Health, 28, 413-433.

Greenwald, P. (1984). Epidemiology: A step forward in the scientific approach to preventing cancer through chemoprevention. Public Health Reports, 99, 259-264.
Hearst, N., Newman, T. B., \& Hulley, S. B. (1986). Delayed effects of the military draft on mortality: A randomized natural experiment. New England Journal of Medicine, 314, 620-624.

Hoge, C. W., Auchterlonie, J. L., \& Milliken, C. S. (2006). Mental health problems, use of mental health services, and attrition from military service after returning from deployment to Iraq or Afghanistan. Journal of the American Medical Association, 295, 1023-1032.

Hoge, C. W., Castro, C. A., Messer, S. C., McGurk, D., Cotting, D. I., \& Koffman, R. L. (2004). Combat duty in Iraq and Afghanistan, mental health problems, and barriers to care. New England Journal of Medicine, 351, 13-22.

Holder, H., Flay, B., Howard, J., Boyd, G., Voas, R., \& Grossman, M. (1999). Phases of alcohol problem prevention research. Alcoholism, Clinical and Experimental Research, 23, 183-194.

Insel, T. R., \& Fenton, W. S. (2005). Psychiatric epidemiology: It's not just about counting anymore. Archives of General Psychiatry, $62,590-592$.

Katon, W., Zatzick, D., Bond, G., \& Williams, J., Jr. (2006). Dissemination of evidence-based mental health interventions: Importance to the trauma field. Journal of Traumatic Stress, 19, 611-623.

Kazdin, A. (2001). Progression of therapy research and clinical application of treatment require better understanding of the change process. Clinical Psychology: Science and Practice, 8, 143-151.

Kessler, R. C., Sonnega, A., Bromet, E., Hughs, M., \& Nelson, C. B. (1995). Posttraumatic stress disorder in the National Comorbidity Survey. Archives of General Psychiatry, 52, 10481060 .

Kulka, R. A., Schlenger, W. E., Fairbank, J. A., Hough, R. L., Jordan, B. K., Marmar, C. R., et al. (1990). Trauma and the Vietnam War generation: Report of findings from the national Vietnam veterans readjustment study (Vol. 18). New York: Brunner/Mazel.

Kundi, M. (2006). Causality and the interpretation of epidemiologic evidence. Environmental Health Perspectives, 114, 969-974.

Litz, B. T., Gray, M. J., Bryant, R. A., \& Adler, A. B. (2002). Early interventions for trauma: Current status and future directions. Clinical Psychology: Science and Practice, 9, 112-134.

Mackenzie, E. J., Rivara, F. P., Jurkovich, G. J., Nathens, A. B., Frey, K. P., Egleston, B. L., et al. (2006). A national evaluation of the effect of trauma-center care on mortality. New England Journal of Medicine, 354, 366-378.

McGaugh, J. L., Introini-Collison, I. B., \& Nagahara, A. H. (1988). Memory-enhancing effects of posttraining naloxone: Involvement of beta-noradrenergic influences in the amygdaloid complex. Brain Research, 446, 37-49. 
Mook, D. G. (1983). In defense of external invalidity. American Psychologist, 38, 379-387.

Morgan, C. A., 3rd, Krystal, J. H., \& Southwick, S. M. (2003). Toward early pharmacological posttraumatic stress intervention. Biological Psychiatry, 53, 834-843.

National Institute of Mental Health. (1999). Bridging science and service: A report by the National Advisory Mental Health Council's Clinical Treatment and Services Research Workgroup (NIH Publication No. 99-4353): Bethesda, MD: Author.

National Institute of Mental Health. (2002). Mental health and mass violence: Evidence-based early psychological intervention for victims/survivors of mass violence. A workshop to reach consensus on best practices (NIH Publication No. 02-5138). Washington, DC: Author.

Norris, F. H., Friedman, M. J., Watson, P. J., Byrne, C. M., Diaz, E., \& Kaniasty, K. (2002). 60,000 disaster victims speak: Parts I and II. An empirical review of the empirical literature, 1981-2001. Psychiatry, 65, 207-260.

Pellmar, T. C., \& Eisenberg, L. (Eds.). (2000). Bridging disciplines in the brain, behavioral, and clinical sciences. Washington, DC: National Academy Press.

Pitman, R. K., \& Delahanty, D. L. (2005). Conceptually driven pharmacologic approaches to acute trauma. CNS Spectrums, 10, 99-106.

Pitman, R. K., Sanders, K. M., Zusman, R. M., Healy, A. R., Cheema, F., Lasko, N. B., et al. (2002). Pilot study of secondary prevention of posttraumatic stress disorder with propranolol. Biological Psychiatry, 51, 189-192.

Reijneveld, S. A., Crone, M. R., Schuller, A. A., Verhulst, F. C., \& Verloove-Vanhorick, S. P. (2005). The changing impact of a severe disaster on the mental health and substance misuse of adolescents: Follow-up of a controlled study. Psychological Medicine, 35, 367-376.

Reijneveld, S. A., Crone, M. R., Verhulst, F. C., \& VerlooveVanhorick, S. P. (2003). The effect of a severe disaster on the mental health of adolescents: A controlled study. Lancet, 362, 691-696.

Rothman, K. J., \& Greenland, S. (1998). Modern epidemiology (2nd ed.). Philadelphia: Lippincott, Williams \& Wilkins.

Rounsaville, B., Carroll, K. M., \& Onken, L. S. (2001). A stage model of behavioral therapies research: Getting started and moving on from stage I. Clinical Psychology: Science and Practice, 8, 133-142.

Sabin, J. A., Zatzick, D. F., Jurkovich, G., \& Rivara, F. P. (2006). Primary care utilization and detection of emotional distress after adolescent traumatic injury: Identifying an unmet need. Pediatrics, 117, 130-138.

Schelling, G., Kilger, E., Roozendaal, B., de Quervain, D. J., Briegel, J., Dagge, A., et al. (2004). Stress doses of hydrocortisone, traumatic memories, and symptoms of posttraumatic stress disorder in patients after cardiac surgery: A randomized study. Biological Psychiatry, 55, 627-633.

Schnurr, P. P., \& Green, B. L. (Eds.). (2004). Trauma and health: Physical health consequences of exposure to extreme stress. Washington, DC: American Psychological Association.

Schoenfeld, F. B., Marmar, C. R., \& Neylan, T. C. (2004). Current concepts in pharmacotherapy for posttraumatic stress disorder. Psychiatric Services, 55, 519-531.

Shrout, P. E. (1998). Causal modeling of epidemiological data on psychiatric disorders. Social Psychiatry and Psychiatric Epidemiology, 33, 400-404.

Simon, G. E., Unutzer, J., Young, B. E., \& Pincus, H. A. (2000). Large medical databases, population-based research, and patient confidentiality. American Journal of Psychiatry, 157, 1731-1737.

Southam-Gerow, M. A., Ringeisen, H. L., \& Sherrill, J. T. (2006). Integrating interventions and services research: Progress and prospects. Clinical Psychology: Science and Practice, 13, 1-8.

Spinazzola, J., Blaustein, M., \& van der Kolk, B. A. (2005). Posttraumatic stress disorder treatment outcome research: The study of unrepresentative samples? Journal of Traumatic Stress, 18, 425436.

Steenland, K., \& Armstrong, B. (2006). An overview of methods for calculating the burden of disease due to specific risk factors. Epidemiology, 17, 512-519.

Stein, M. (in press). Pharmacoprevention of adverse psychiatric sequelae of physical injury. Journal of Traumatic Stress.

Street, L. L., Niederehe, G., \& Lebowitz, B. D. (2000). Toward greater public health relevance for psychotherapeutic intervention research: A NIMH workshop report. Clinical Psychology: Science and Practice, 7, 127-137.

Susser, M. (1991). What is a cause and how do we know one? A grammar for pragmatic epidemiology. American Journal of Epidemiology, 133, 635-648.

Susser, M. (1997). Steps toward discovering causes: Divergence and convergence of epidemiology and clinical medicine. Epidemiologia e Prevenzione, 21, 160-168.

Ursano, R. J., Bell, C., Eth, S., Friedman, M., Norwood, A., Pfefferbaum, B., et al. (2004). Practice guideline for the treatment of patients with acute stress disorder and posttraumatic stress disorder. American Journal of Psychiatry, 161(Suppl. 11), 3-31. 
Vaiva, G., Ducrocq, F., Jezequel, K., Averland, B., Lestavel, P., Brunet, A., et al. (2003). Immediate treatment with propranolol decreases posttraumatic stress disorder two months after trauma. Biological Psychiatry, 54, 947-949.

Vlahov, D., Galea, S., Ahern, J., Resnick, H., Boscarino, J. A., Gold, J., et al. (2004). Consumption of cigarettes, alcohol, and marijuana among New York City residents six months after the September 11 terrorist attacks. American Journal of Drug and Alcohol Abuse, 30, 385-407.

Vlahov, D., Galea, S., Ahern, J., Resnick, H., \& Kilpatrick, D. (2004). Sustained increased consumption of cigarettes, alcohol, and marijuana among Manhattan residents after September 11, 2001. American Journal of Public Health, 94, 253254.

Vlahov, D., Galea, S., Ahern, J., Rudenstine, S., Resnick, H., Kilpatrick, D., et al. (2006). Alcohol drinking problems among New York City residents after the September 11 terrorist attacks. Substance Use and Misuse, 41, 1295-1311.

Wang, P. S., Lane, M., Olfson, M., Pincus, H. A., Wells, K. B., \& Kessler, R. C. (2005). Twelve-month use of mental health services in the United States: Results from the National Comorbidity Survey Replication. Archives of General Psychiatry, 62, 629-640.

Weisz, J. R., Chu, B. C., \& Polo, A. J. (2004). Treatment dissemination and evidence-based practice: Strengthening intervention through clinician-researcher collaboration. Clinical Psychology: Science and Practice, 11, 300-307.

Zatzick, D., Grossman, D. C., Russo, J., Pynoos, R., Berliner, L., Jurkovich, G., et al. (2006). Predicting posttraumatic stress symptoms longitudinally in a representative sample of hospitalized injured adolescents. Journal of the American Academy of Child and Adolescent Psychiatry, 45, 1188-1195.
Zatzick, D., Kang, S., Kim, S., Leigh, P., Kravitz, R., Drake, C., et al. (2000). Patients with recognized psychiatric disorders in trauma surgery: Incidence, inpatient length of stay, and cost. Journal of Trauma, 49, 487-495.

Zatzick, D., Kang, S. M., Muller, H. G., Russo, J. E., Rivara, F. P., Katon, W., et al. (2002). Predicting posttraumatic distress in hospitalized trauma survivors with acute injuries. American Journal of Psychiatry, 159, 941-946.

Zatzick, D., \& Roy-Byrne, P. (2006). From bedside to bench: How the epidemiology of clinical practice can inform the secondary prevention of PTSD. Psychiatric Services, 57, 1726-1730.

Zatzick, D., Roy-Byrne, P., Russo, J., Rivara, F., Droesch, R., Wagner, A., et al. (2004). A randomized effectiveness trial of stepped collaborative care for acutely injured trauma survivors. Archives of General Psychiatry, 61, 498-506.

Zatzick, D., Russo, J., Rajotte, E., Uehara, E., Roy-Byrne, P., Ghesquiere, A., et al. (in press). Strengthening the patientprovider relationship in the aftermath of physical trauma through an understanding of the nature and severity of posttaumatic concerns. Psychiatry.

Zatzick, D., Russo, J., Rivara, F., Roy-Byrne, P., Jurkovich, G., \& Katon, W. (2005). The detection and treatment of posttraumatic distress and substance intoxication in the acute care inpatient setting. General Hospital Psychiatry, 27(1), 57-62.

Zatzick, D., Simon, G. E., \& Wagner, A. W. (2006). Developing and implementing randomized effectiveness trials in general medical settings. Clinical Psychology: Science and Practice, 13, 53-68.

Zatzick, D. F., Rivara, F. P., Nathens, A. B., Jurkovich, G. J., Wang, J., Fan, M. Y., et al. (2007) (in press). A nationwide US study of posttraumatic stress symptoms after hospitalization for physical injury. Manuscript submitted for publication. 